\title{
ECOTOXICIDAD ACUÁTICA DE DOS COLORANTES Y DE TRES ANTIPARASITARIOS DE IMPORTANCIA EN ACUICULTURA EN Daphnia magna
}

\section{AQUATIC ECOTOXICITY OF TWO DYES AND THREE ANTIPARASITICS OF IMPORTANCE IN AQUACULTURE ON EMPLOYING Daphnia magna}

\author{
José Iannacone ${ }^{1,2}$ y Lorena Alvariño ${ }^{2}$
}

\section{Resumen}

La presencia y la evaluación ecotoxicológica de productos farmacéuticos en el ambiente acuático es un área de investigación emergente a nivel global. El objetivo del presente trabajo fue evaluar el efecto toxicológico acuático de dos colorantes de importancia en la acuicultura, el azul de metileno y el verde de malaquita, y de tres antiparasitarios antihelmínticos, dos productos naturales Parasin ${ }^{\circledR}$, leche de ojé ${ }^{\circledR}$ y el químico sintético albendazol-praziquantel sobre Daphnia magna Strauss 1820 (Crustácea: Daphniidae). En D. magna ( $\mathrm{CL}_{50}$ a 96 h de exposición), los mayores efectos se encontraron con el azul de metileno $\left(\mathrm{CL}_{50}=0.38 \mathrm{mg} \cdot \mathrm{L}^{-1}\right)$ en comparación al verde de malaquita $\left(\mathrm{CL}_{50}=2.97 \mathrm{mg} \cdot \mathrm{L}^{-1}\right)$. Con relación a los productos antiparasitarios en $D$. magna $\left(\mathrm{CL}_{50}\right.$ a $\left.48 \mathrm{~h}\right)$, los mayores efectos se encontraron con la leche de ojé $\left(\mathrm{CL}_{50}=0.07 \%\right)$ en comparación al Parasin $\left(\mathrm{CL}_{50}=0.99 \%\right)$. Se observó que el praziquantel $\left(\mathrm{CL}_{50}=30.07 \mathrm{mg} \cdot \mathrm{L}^{-1}\right)$ y el albendazol $\left(\mathrm{CL}_{50}=180.4 \mathrm{mg} \cdot \mathrm{L}^{-1}\right)$ produjeron efectos sobre D. magna a $48 \mathrm{~h}$ de exposición. Los dos colorantes produjeron altos efectos en términos de $\mathrm{CL}_{50}$ sobre $D$. magna en relación a las concentraciones requeridas para su uso en acuicultura. Los dos productos naturales antiparasitarios ocasionaron riesgos en el ambiente acuático a concentraciones menores al 1\%. La técnica del cociente de riesgo indicó que el praziquantel y el albendazol no ocasionaron riesgo al ambiente acuático.

Palabras claves: albendazol, azul de metileno, ecotoxicología, praziquantel, pulga de agua, verde de malaquita

\begin{abstract}
Ecotoxicological assessment of pharmaceutical products in aquatic environments is an area of emerging research at global level. The aims of the current research were to evaluate aquatic toxicological effects of two aquaculture important dyes: methylene blue and malachite green, of three anthelminthic antiparasitic products, of two natural products Parasin ${ }^{\circledR}$ and leche de ojé®, and of the synthetic chemical albendazole-praziquantel on Daphnia magna Strauss 1820 (Crustacea: Daphniidae). On D. magna ( $\mathrm{LC}_{50}$ at $96 \mathrm{~h}$ exposure), the highest effects were found with methylene blue $\left(\mathrm{LC}_{50}=0.38 \mathrm{mg} \cdot \mathrm{L}^{-1}\right)$ in comparison to malachite green $\left(\mathrm{LC}_{50}=2.97 \mathrm{mg} \cdot \mathrm{L}^{-1}\right)$. In relation to antiparasitic products on $D$. magna $\left(\mathrm{LC}_{50}\right.$ at $48 \mathrm{~h}$ ), the highest effects were found with leche de ojé $\left(\mathrm{LC}_{50}=0.07 \%\right)$ in comparison to Parasin $\left(\mathrm{LC}_{50}=0.99 \%\right)$. Praziquantel $\left(\mathrm{LC}_{50}=\right.$ $30.07 \mathrm{mg} \cdot \mathrm{L}^{-1}$ ) and albendazole $\left(\mathrm{LC}_{50}=180.4 \mathrm{mg} \cdot \mathrm{L}^{-1}\right)$ were observe to produce effects on $D$. magna at $48 \mathrm{~h}$ of exposure. Both dyes produced high effects in terms of $\mathrm{LC}_{50}$ on $D$. magna in relation to concentrations required for their use in aquaculture. Both antiparasitic natural products produced risks in aquatic environments at concentrations less than $1 \%$. The quotient risk technique indicated that praziquantel and albendazole did not produce risks on an aquatic environment.

Key words: albendazole, methylene blue, ecotoxicology, praziquantel, water flea, malaquite green
\end{abstract}

\section{Introducción}

En la actualidad muchos productos químicos farmacéuticos son producidos y usados en grandes volúmenes a nivel mundial. Por ende, la presencia y la evaluación de los productos farmacéuticos en el ambiente acuático es un área de investigación emergente a nivel global (Halling-Sorensen et al., 1998; Daughton \& Ternes, 1999; Chapman, 2006; Jjemba, 2006). En Europa, aproximadamente 13500 tn de antibióticos son consumidos por año, de los cuales $65 \%$ son usados en medicina humana y $35 \%$ en medicina veterinaria. Una cantidad desconocida de estos componentes son evacuados al ambiente vía las aguas residuales o cuando las heces son usadas con residuos farmacéticos para la fertilización de tierras agrícolas (Lützhoft et al., 1999; Backhaus et al., 2000; Lalumera et al., 2004; Woodward, 2006). Los productos veterinarios y humanos aplicados con propósitos medicinales o como aditivos pueden llegar al ambiente acuático por rutas directas o indirectas (Boxall et al., 2003a b; Boxall \& Long, 2005). 
Muchos productos antihelmínticos como el praziquantel y el albendazol son usados en grandes cantidades y tienen alta potencialidad de ingresar al ambiente (Oh et al., 2006). Sin embargo, no se encuentran bien caracterizadas las consecuencias ecológicas de su ingreso al ambiente acuático, a pesar de su extremadamente baja constante de Henry, que indica la partición de equilibrio entre el aire y el agua, y su razonable solubilidad en agua que sugieren que estos compuestos pueden causar problemas en la columna de agua (Boxall et al., 2003a).

El praziquantel es una de las drogas más usadas para el tratamiento de schistosomiasis producido por Schistosoma mansoni Sambon 1907. El mecanismo exacto de acción del praziquantel no está exactamente entendido en este parásito, pero parece que permite que el parásito sea más susceptible a su eliminación por el sistema inmunológico del hospedero (Karanja et al., 1998). El praziquantel es un tenicida y tiene acción sobre Dipylidium caninum (Linnaeus 1758), larvas de Taenia solium Linnaeus 1758 y Taenia pisiformis Bloch 1780, provocando la pérdida del calcio intracelular y por contracciones masivas, vacuolización y desintegración del helminto (Pareja et al., 2002; Sibat \& Ibañez, 2003; Holm-Martin et al., 2005). El praziquantel es un antihelmíntico de amplio rango en mamíferos, incluyendo al ser humano, que modula la permeabilidad de la membrana celular de los céstodes y tremátodes, permitiendo la desintegración del tegumento (Hempel et al., 2006). También, quimioterapias con praziquantel se han usado para el control de monogeneos en peces (Kim et al., 2001).

Belkind-Valdovinos et al. (2004) indican que el albendazol es empleado para el control de diversas enteroparasitosis humana causada por nemátodos en áreas endémicas del Perú. El tratamiento con albendazol es también eficiente para el control de nemátodos intestinales de animales domésticos (Leathwick et al., 2006). Alvarez-Sánchez et al. (2006) señalan que el albendazol es eficiente para el control de Fasciola hepatica Linnaeus 1758 en animales de importancia pecuaria.

En el Perú, existen muchas plantas con propiedades antiparasitarias individuales y en mezclas de importancia etnobotánica que no han sido evaluadas con relación a su impacto en el ambiente acuático (Maguiña \& Iannacone, 2000).

El ojé (Ficus insipida Willd; Moaceae) produce un látex con propiedades antihelmínticas y es aún usado en la medicina tradicional por la gente local e indígena de las regiones amazónicas (Hansson et al., 2005). Varias sustancias están ligadas con su efectividad: ficina, filoxantina, b-amirina, lepeol, lavandulol, phyllantel, eloxantina, filantelol y doxantina. Sin embargo, este vermífugo debido a su alta toxicidad aguda ha producido varios casos de intoxicación por sobredosis en el ser humano (De Amorin et al., 1999).
La semilla del zapallo (Cucurbita maxima Dutch; Cucurbitaceae) es usada en el Perú en medicina tradicional ya que presenta propiedades antiparasitarias sobre céstodos (Guarrera, 1999; Díaz et al., 2004) y sobre protozoos causantes de la malaria (Amorim et al., 1991). Se han descrito varias sustancias químicas relacionadas con su efecto antihelmíntico como cucurbitina, peporesina, lecitina, guanosina, titosterina y el ácido citrúlico.

Las hojas de la hierba buena (Mentha spicata (Briq.) Harley; Labiatae) es una planta aromática y medicinal usada por el hombre moderno (Lagarto et al., 1999). Estudios etnobótanicos informan de su empleo como antiséptico debido a sus aceites esenciales (Imai et al., 2001). Se le usa, además, como tóxico y repelente del vector de malaria Anopheles stephensi Liston 1901 (Tripathi et al., 2004). Se ha evaluado su acción antimutagénica (Yu et al., 2004) y sus efectos en la biología reproductiva humana (Akdogan et al., 2007).

Varias medicinas veterinarias pueden ser usadas como antisépticos en la acuicultura, como es el caso del verde de malaquita y del azul de metileno, y por ende muchos organismos acuáticos no destinatarios del control son expuestos a estas sustancias químicas (Wollenberg et al., 2000; Christensen et al., 2006; Hernando et al., 2007).

El colorante verde de malaquita es usado globalmente como un antifúngico y un antiparasitario en crustáceos y peces de importancia en acuicultura (Rintamaki et al., 2005; Zilberg \& Tamar, 2006). Se le ha empleado para el control del protozoo Ichthyopthirius multifiliis Bouquet 1876 (Ichthyoptariasis), y del monogeneo Dactylogyrus vastator Nybelin 1924 (Schmahl et al., 1992; Molnar, 1995; Rintamaki et al., 2005). Su uso no está permitido en los Estados Unidos, la Unión Europea y en algunos países del sudeste Asiático como Tailandia, debido a que se considera que tiene un rol altamente tóxico sobre las enzimas respiratorias. En adición, es esencial tener periodos largos de carencia después de la aplicación del verde de malaquita debido a los residuos persistentes en los alimentos de origen acuático (GESAMP, 1997; Grant et al., 2001; Andersen et al., 2006). También el verde de malaquita es empleado en salud, en la industria de alimentos y textil; así como para el control de helmintiasis en peces y otros organismos acuáticos (Van de Riet et al., 2005; Hernando et al., 2007). Se le ha reportado que causa variación de los parámetros hematológicos, carcinogénesis, mutagénesis, fracturas cromosómicas, teratogenicidad y toxicidad respiratoria (Silveira et al., 2004; Srivastava et al., 2004).

El azul de metileno es ocasionalmente usado como antiséptico tipo antifúngico e infecciones por protozoos en cultivo de peces. Puede proveer protección contra la intoxicación por nitrilos (GESAMP, 1997). El azul de metileno es usado como 
un fijador y colorante básico para el diagnóstico de protozoos parásitos, células sanguíneas y otras preparaciones microscópicas (Iannacone et al., 1999; Costamagna et al., 2004). Stockert \& Herkovits (2003) han evaluado la toxicidad del azul de metileno sobre embriones de Bufo arenarum (Hensel 1867). Rifici et al. (1996) han determinado la toxicidad sobre el pez Pimephales promelas Rafinesque 1820. En Salud Pública al azul de metileno se le usa para el tratamiento de una enfermedad denominada metahemoglobinemia (Clifton \& Leikin, 2003; Stocche et al., 2004) y para casos de intoxicación por herbicidas (Watt et al., 2005). Tambien se ha evaluado al carbón activado y a la planta acuática Spirodela polyrrhiza (L.) Schleid como agentes de remoción del azul de metileno en el agua (Waranusantigui et al., 2003; Moreno et al., 2006).

Daphnia magna Strauss 1820 (Crustácea: Daphniidae) es una especie partenogenética usada extensivamente en pruebas de toxicidad para evaluar sustancias químicas puras, aguas residuales domésticas e industriales, aguas superficiales o subterráneas, agua potable, y lixiviados entre otros. Esto es debido a: 1) amplia distribución geográfica, 2) importante papel en la comunidad zooplanctónica, 3) facilidad de cultivo, 4) corto ciclo de vida con la producción de un alto número de crías (Castillo, 2004; Kallqvist et al., 2006; Reynaldi et al., 2006).

Muchos productos farmacéutico aplicados con propósitos medicinales o como aditivos pueden llegar finalmente al ambiente dulceacuícola por rutas directas 0 indirectas. Entre dichos compuestos tenemos a productos antihelmínticos como antiparasitarios naturales a base de plantas y a varias medicinas veterinarias que pueden ser usadas como antifúngicos y antiparasitarios en la acuicultura. Sin embargo, no se conocen las consecuencias ecológicas del ingreso de estas sustancias a la columna de agua. Por ende, la evaluación del efecto de estos compuestos en organismos acuáticos no destinatarios, como el cladócero $D$. magna, a través de una evaluación del riesgo ecológico permitirá tomar las medidas de mitigación necesarias para la protección de los ambientes acuáticos por productos farmacéuticos.

Por ende, el objetivo del presente trabajo fue evaluar la ecotoxicidad acuática de dos antifúngicos usados en acuicultura y de tres antiparasitarios empleando $D$. magna como un organismo de ensayo.

\section{Materiales y métodos}

Los bioensayos ecotoxicológicos acuáticos se realizaron en el Laboratorio de Invertebrados, Facultad de Ciencias Biológicas, Universidad Ricardo Palma, Santiago de Surco, Lima, Perú.

\section{Productos químicos \\ Colorantes}

Verde de malaquita: $\left(\mathrm{C}_{48} \mathrm{H}_{50} \mathrm{~N}_{4} \mathrm{O}_{4} \cdot 2 \mathrm{HC}_{2} \mathrm{O}_{4}\right)$, CAS = 569-64-2 y una solubilidad en agua de $20 \mathrm{~g} \mathrm{~L}^{-1}$. Se preparó una solución madre a una concentración de 40 $\mathrm{mg} \mathrm{L}^{-1}$. A partir de estas soluciones se realizaron las diluciones respectivas empleando el medio de cultivo artificial de Daphnia (Medio ADaM) (Iannacone et al., 2007). Se utilizaron botellas plásticas de un litro para la preparación de cada una de las cinco concentraciones. Para el verde de malaquita se utilizó las siguientes cinco concentraciones en orden creciente: 2,5, 5, 10, 20 y $40 \mathrm{mg} \mathrm{L}^{-1}$.

Azul de metileno: $\left(\mathrm{C}_{16} \mathrm{H}_{18} \mathrm{C}_{\mathrm{l}} \mathrm{N}_{3} \mathrm{~S} \cdot 3 \mathrm{H}_{2} \mathrm{O}\right)$. CAS = 61-73-4 y una solubilidad en agua de $40 \mathrm{~g} \mathrm{~L}^{-1}$. Se siguió el mismo procedimiento que para el verde de malaquita, pero se preparó la solución madre a $20 \mathrm{mg}$ $\mathrm{L}^{-1}$. Se emplearon las siguientes cinco concentraciones en orden creciente: $0.156,0.312,0.625,1.25$ y 2.5 $\mathrm{mg} \mathrm{L}^{-1}$.

\section{Antiparasitarios}

Parasin ${ }^{\circledR}$ : El producto es elaborado por el laboratorio Bionaturista ${ }^{\circledR}$. Este producto es un macerado natural. El antiparasitario está compuesto por semillas de zapallo C. maxima, semillas de papaya Carica papaya L., hierba buena (M. spicata), propoleo, ceras, polen y aceites aromáticos, entre otros elementos. Este producto tiene acción antihelmíntica y produce alteraciones en la motilidad del helminto, proteólisis y muerte del mismo, y microscópicamente provoca alteración del tegumento, de la membrana basal, estructuras internas y ruptura de la cáscara de los huevos en los proglótidos maduros y grávidos de los céstodos. El screening fitoquímico fue realizado en el Laboratorio de análisis químico de la Pontificia Universidad Católica del Perú (PUCP). Los procedimientos estándares para la marcha fitoquímica mostraron para saponinas (producción de espuma) = -; taninos $\left(\right.$ Gelatina/ $\left.\mathrm{FeCl}_{3}\right)=+$; flavonoides (Shinoda) $=$ -; esteroides (Libermann B) = -; quinonas (Bornträger) $=\quad-;$ fenoles $($ Rosenheim $)=-;$ alcaloides (Dragendorff/Mayer) $=+\mathrm{y}$ aminoácidos (Ninhidrina) $=+$. Siendo $-=$ reacción negativa $\mathrm{y}+=$ reacción positiva.

Leche de Ojéß: Este antiparasitario es elaborado por el laboratorio Santa Natura ${ }^{\circledR}$. Este producto es un macerado compuesto por Leche de Ojé F. insipida, hierba buena y miel. En su mecanismo de acción disuelve las proteínas de la cutícula que recubre el cuerpo de los parásitos intestinales, permitiendo su mortalidad y digestión por los mecanismos bioquímicos gastrointestinales normales (Giraldo et al., 2006). El screening fitoquímico fue realizado en el Laboratorio de análisis químico de la Pontificia Universidad Católica del Perú (PUCP). Los procedimientos estándares para la marcha fitoquímica mostraron para saponinas (producción de espuma) = -; taninos $\left(\right.$ Gelatina $\left./ \mathrm{FeCl}_{3}\right)$ = -; flavonoides (Shinoda) = -; 
esteroides (Libermann B) = -; quinonas (Bornträger) = -; fenoles (Rosenheim) = -; alcaloides (Dragendorff/Mayer) $=+\mathrm{y}$ aminoácidos (Ninhidrina) $=+$.

Tanto para el Parasín y para la Leche de ojé, se emplearon las siguientes cinco concentraciones a partir del producto comercial, los cuales se diluyeron en el medio ADaM: $0.156 \%, 0.313 \%, 0.625 \%, 1.25 \%$ y $2.5 \%$.

Quantel-Plus®: Producto elaborado por los laboratorios Farmindustria ${ }^{\circledR}$. Es un comprimido compuesto por dos ingredientes activos (ia): praziquantel (50 mg) y albendazol (300 mg). El praziquantel tiene un CAS de 55268-74-1 y su solubilidad en agua es de $400 \mathrm{mg} \mathrm{L}^{-1}$. El albendazol tiene un CAS 54965-21-8 y una solubilidad en agua de $0.42 \mathrm{~g} \mathrm{~L}^{-1}$. Ambos actúan como antihelmínticos, causando la disrupción del metabolismo del helminto, incluyendo la disminución de energía, que inmoviliza y posteriormente mata el helminto sensible. Para el praziquantel se utilizó las siguientes cinco concentraciones en orden creciente: 15.63, 31.25, 62.5, 125 y $250 \mathrm{mg} \mathrm{L}^{-1}$. En cambio, para el albendazol se usó las siguientes cinco concentraciones en orden creciente: $93.75,187.5$, 375, 750 y $1500 \mathrm{mg} \mathrm{L}^{-1}$, los cuales se diluyeron en el medio ADaM.

\section{Organismo prueba \\ Daphnia magna}

Hembras adultas de esta especie se obtuvieron del acuario “Cleo” procedente del distrito de Lince, Lima, Perú, y se llevaron al laboratorio en recipientes plásticos de 2 L de capacidad. Hembras partenogenéticas se colocaron en el medio nutritivo ADaM. La preparación del medio se realizó de la siguiente forma: 9.9 g de sales obtenidas por evaporación del agua de mar se adicionaron a $60 \mathrm{~L}$ de agua de grifo declorinada, reposada y hiperoxigenada durante $24 \mathrm{~h}$. Luego se agregó $138 \mathrm{~mL}$ de una solución de cloruro de calcio $\left(117.6 \mathrm{~g} \cdot \mathrm{L}^{-1}\right), 132 \mathrm{~mL}$ de una solución de bicarbonato de sodio $\left(25.2 \mathrm{~g} \cdot \mathrm{L}^{-1}\right)$ y $6 \mathrm{~mL}$ de una solución de Oxido de Selenio (0.07 $\mathrm{g} \mathrm{L}^{-1}$ ) (Klüttgen et al., 1994). Los cultivos parciales se mantuvieron a una temperatura de $21 \pm 2^{\circ} \mathrm{C}$ y a un fotoperiodo aproximadamente de 12:12. El oxígeno disuelto tuvo una concentración sobre $6 \mathrm{mg} \cdot \mathrm{L}^{-1}$ (Castillo, 2004). Para el desarrollo de la prueba de toxicidad aguda con $D$. magna se empleó cohortes de neonatos ( $<24 \mathrm{~h}$ de nacidos). La duración total de la prueba fue de $48 \mathrm{~h}$ de exposición para los antiparasitarios y para los dos colorantes. En adición, para los dos colorantes se dieron lecturas a $96 \mathrm{~h}$. Se empleó un factor de dilución de 0.5. A cada envase circular de $250 \mathrm{~mL}$ se procedió a agregar $100 \mathrm{~mL}$ de cada una de las concentraciones de las sustancias químicas empleadas, a los que se transfirieron diez neonatos de D. magna. Se usó como criterio de mortalidad la carencia de movilidad o la ausencia de ritmo cardiaco a 15 s de observación al microscopio estereoscopio. Antes de efectuar las lecturas se agitó los envases en forma circular para reactivar el movimiento de los organismos que se posaban inmóviles en el fondo (Castillo, 2004).

\section{Análisis de datos}

Las valores de $\mathrm{CL}_{50}$ en $\mathrm{mg} \cdot \mathrm{L}^{-1}$ para el verde malaquita y para el azul de metileno de otras especies de crustáceos acuáticos fue obtenido de una base de datos electrónica (http://www.pesticideinfo.org/Detail_Chemical.jsp). Las pruebas de toxicidad de los antiparasitarios y de los colorantes de importancia en la acuicultura sobre $D$. magna se evaluaron en cinco concentraciones más el control en el medio nutritivo ADaM, con cuatro repeticiones, en un diseño de Bloque Completo al Azar (DBCA): 6 x 4. La eficacia de los tratamientos y las repeticiones se evaluó a través de un análisis de varianza (ANDEVA) de dos vías, previa transformación de los datos a raíz cuadrada del arcoseno. En el caso de existir diferencias significativas entre los tratamientos y entre las repeticiones se realizó la prueba de Tukey. Los cálculos de la mortalidad corregida se realizaron mediante la fórmula de Abbott en caso de muerte natural en el grupo testigo cuando fue menor al $20 \%$ (Macedo et al., 1997). Las CL(E) $\mathrm{S}_{50}$ se calcularon usando el programa computarizado Probit versión 1.5. El modelo de regresión fue verificado usando el estadístico Chi-cuadrado. Los resultados para los estadísticos descriptivos e inferenciales se analizaron mediante el paquete estadístico SPSS versión 15,0.

\section{Resultados \\ Colorantes}

Se observó un incremento en los valores de $\mathrm{CL}_{50}$ en $\mathrm{mg} \cdot \mathrm{L}^{-1}$ para el verde de malaquita y para el azul de metileno a 24 h, 48 h, 72 h y 96 h de exposición sobre D. magna (Figura 1). Para el verde de malaquita, el valor de $\mathrm{CL}_{50}$ a $24 \mathrm{~h}$ fue $9.27 \mathrm{mg} \cdot \mathrm{L}^{-1}$, estadísticamente diferente a los valores de $48 \mathrm{~h}\left(\mathrm{CL}_{50}=4.65 \mathrm{mg} \cdot \mathrm{L}^{-1}\right)$, $72 \mathrm{~h}\left(\mathrm{CL}_{50}=3,23 \mathrm{mg} \cdot \mathrm{L}^{-1}\right)$ y $96 \mathrm{~h}$ de exposición $\left(\mathrm{CL}_{50}\right.$ $\left.=2.97 \mathrm{mg} \cdot \mathrm{L}^{-1}\right) \quad(\mathrm{F}=32.61 ; \mathrm{P}=0.000)($ Figura 1$)$. Para el azul de metileno, el valor de $\mathrm{CL}_{50}$ a $24 \mathrm{~h}$ fue 2.37 $\mathrm{mg} \cdot \mathrm{L}^{-1}$, estadísticamente diferente al valor de $48 \mathrm{~h}$ de exposición $\left(\mathrm{CL}_{50}=1.20 \mathrm{mg} \cdot \mathrm{L}^{-1}\right)$, siendo a su vez este último diferente al de $72 \mathrm{~h}\left(\mathrm{CL}_{50}=0.54 \mathrm{mg} \cdot \mathrm{L}^{-1}\right)$ y $96 \mathrm{~h}$ de exposición $\left(\mathrm{CL}_{50}=0,38 \mathrm{mg} \cdot \mathrm{L}^{-1}\right)$ sobre $D$. magna $(\mathrm{F}=53.83 ; \mathrm{P}=0.000$ ) (Figura 1$)$. Para todos los periodos de exposición, existieron diferencias estadísticamente significativas entre los valores de $\mathrm{CL}_{50}$ para el verde de malaquita y para el azul de metileno a las $24 \mathrm{~h}(\mathrm{t}=7.12 ; \mathrm{P}=0.000)$, a las $48 \mathrm{~h}(\mathrm{t}=$ 10.09; $\mathrm{P}=0.000)$, a las $72 \mathrm{~h}(\mathrm{t}=12.61 ; \mathrm{P}=0.000)$ y a las $96 \mathrm{~h}$ de exposición sobre $D$. magna $(\mathrm{t}=25.16$; $\mathrm{P}=0.000)$. 


\section{Antiparasitarios}

Los porcentajes de mortalidad de $D$. magna a las cinco concentraciones evaluadas para los productos antiparasitarios comerciales: Parasín y leche de ojé son indicados en la Figura 2. Los valores de $\mathrm{CL}_{50}$ y los límites de confianza superior e inferior fueron $1.34 \%$ $(0.81 \%-2.04 \%)$ y $0.99 \%(0.67 \%-1.20 \%)$ para parasin, y $0.53 \%$ (0.31 \%- $0.79 \%$ ) y $0.07 \%$ (0.01\% - $0.12 \%$ para leche de ojé a 24 h y 48 h de exposición, respectivamente. La leche de ojé presentó mayor toxicidad según los valores de $\mathrm{CL}_{50}$ a $24 \mathrm{~h}$ de exposición y a 48 h de exposición que el producto comercial Parasín. Las Figuras 3 y 4 nos muestran que existen diferencias en los valores de $\mathrm{CL}_{50}$ para el praziquantel $\left(\mathrm{CL}_{50-24 \mathrm{~h}}=79.80 \mathrm{mg} \cdot \mathrm{L}^{-1} ; \mathrm{CL}_{50-48 \mathrm{~h}}=30.07\right.$ $\left.\mathrm{mg} \cdot \mathrm{L}^{-1}\right)$ y para el albendazol $\left(\mathrm{CL}_{50-24 \mathrm{~h}}=478.6 \mathrm{mg} \cdot \mathrm{L}^{-1}\right.$; $\mathrm{CL}_{50-48 \mathrm{~h}}=180.4 \mathrm{mg} \cdot \mathrm{L}^{-1}$ ) a $24 \mathrm{~h}$ y a $48 \mathrm{~h}$ de exposición, respectivamente.

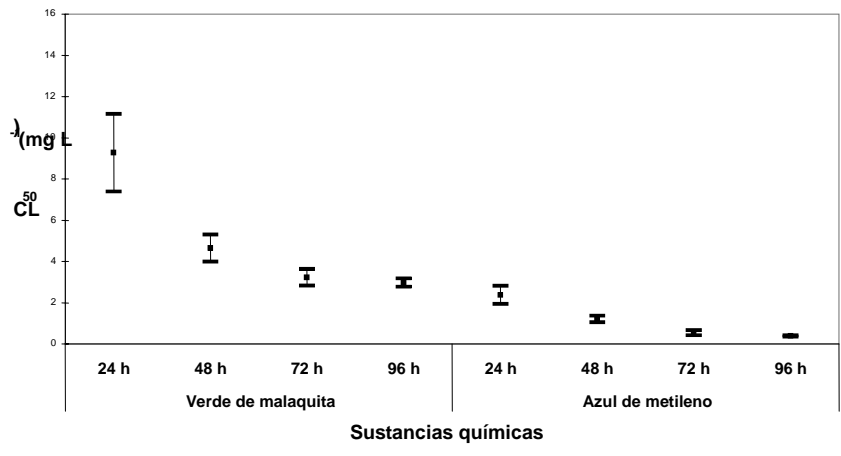

Figura 1. Valores de CL50 del verde de malaquita y del azul de metileno sobre Daphnia magna entre $24 \mathrm{~h}$ y 96 h de exposición.

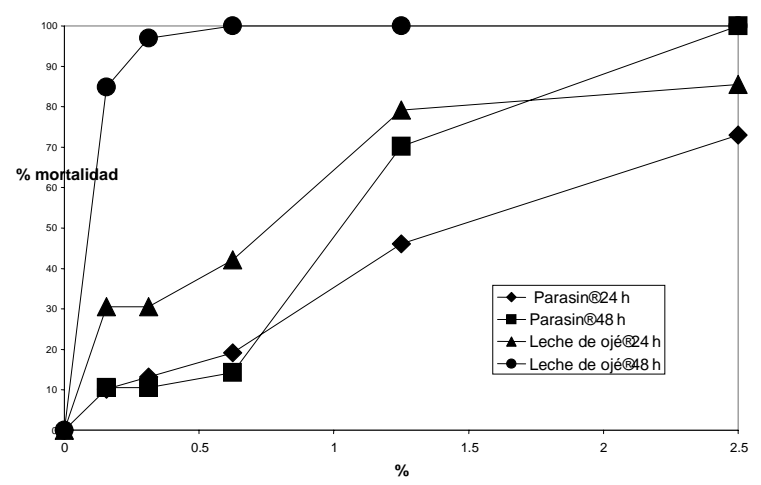

Figura 2. Curvas de mortalidad de Daphnia magna por acción del Parasin y de la Leche de ojé.

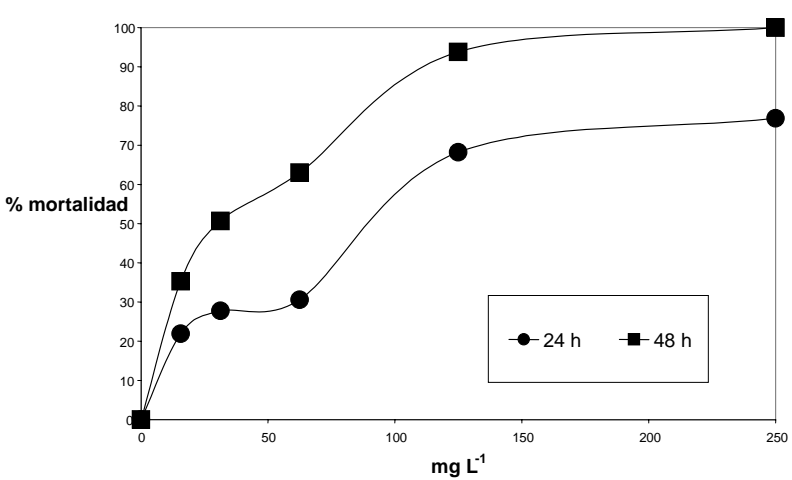

Figura 3. Curvas de mortalidad de Daphnia magna por acción del Praziquantel.

\section{Discusión \\ Colorantes}

Para el caso del verde de malaquita, un análisis comparativo con 40 valores de $\mathrm{CL}_{50}$ para 17 especies diferentes de crustáceos acuáticos, ubica a la pulga de agua $D$. magna en la posición 25 , siendo la décima especie más sensible al verde de malaquita (Tabla 1 ). El valor de $\mathrm{CL}_{50}$ de 96 h de $D$. magna es más tóxico que la $\mathrm{CL}_{50}$ de $10 \mathrm{mg} \cdot \mathrm{L}^{-1}$ del protozoario parásito apicomplexa Perkinsus marinus (Mackin, Owen and Collier 1950) Levine 1978 de la ostra Crassostrea virginica Gmelin 1791 (http://www.pesticideinfo.org/Detail_Chemical.jsp) y del monogeneo $D$. vastator en Cyprinus carpio (Molnar, 1995). Sin embargo, recomendaciones de su uso como antiséptico en peces, lo recomiendan en baños de 0.10 a $0.15 \mathrm{mg} \cdot \mathrm{L}^{-1}$ de verde de malaquita, por lo que bajo estas condiciones no se estaría produciendo ningún riesgo para el ambiente. En adición, se han observado en peces para el verde de malaquita valores de toxicidad que fluctúan entre 0.066 a $1 \mathrm{mg} \cdot \mathrm{L}^{-1}$, siendo Clarias macrocephalus Günther 1864 la especie más sensible y Heteropneustes fossilis (Bloch 1794) la menos sensible entre cinco especies de peces (http://www.pesticideinfo.org/Detail_Chemical.jsp). El verde de malaquita a $6 \mathrm{mgL}^{-1}$ no produjo ningún efecto significativo sobre la bacteria Vibrio fischeri (Beijerinck 1889) (Hernando et al., 2007). El uso del verde de malaquita no está permitido en varios países del mundo, debido a que se considera que tiene un efecto altamente tóxico, y debido a los residuos persistentes en el ambiente acuático al emplearse en terapias de inmersión (GESAMP, 1997; Andersen et al., 2006). Por ende, Schmahl et al. (1992) recomiendan que, para reducir el riesgo de las terapias de inmersión por verde de malaquita en peces ornamentales como Paracheirodon axelrodi (Schultz 
1956), la medicación debe ser a través de la alimentación.

Para el caso del azul de metileno, el análisis comparativo con 6 valores de $\mathrm{CL}_{50}$ para tres especies diferentes de crustáceos, ubica a la pulga de agua $D$. magna en la posición primera en términos de sensibilidad (Tabla 2). En adición, se han observado en peces para el azul de metileno valores de toxicidad que fluctuán entre 1 a $188 \mathrm{mg} \cdot \mathrm{L}^{-1}$, siendo Morone saxatilis (Walbaum 1792) el pez mas sensible y $H$. fossilis el menos sensible entre 10 especies ícticas (http://www.pesticideinfo.org/Detail_Chemical.jsp). Se ha registrado la presencia de serios problemas de contaminación acuática por el azul de metileno al reducir la penetración de la luz y la fotosíntesis (Waranusantigui et al., 2003).

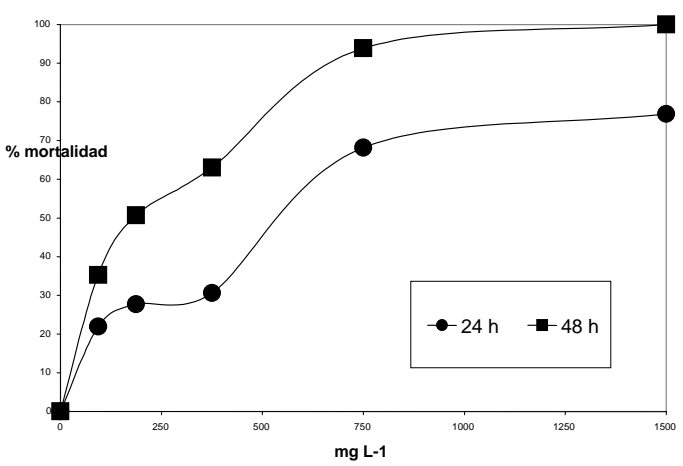

Figura 4. Curvas de mortalidad de Daphnia magna por acción del albendazole.

Tabla 2. Valores comparativos de $\mathrm{CL}_{50}$ con especies de crustáceos acuáticos expuestos al azul de metileno.

\begin{tabular}{lccc}
\hline \multicolumn{1}{c}{ Especie de crustáceo } & $\begin{array}{c}\mathrm{CL}_{50} \\
\left(\mathrm{mg} \mathrm{L}^{-1}\right)\end{array}$ & $\begin{array}{c}\text { Tiempo de } \\
\text { exposición } \\
(\mathrm{h})\end{array}$ & Estadio \\
\hline $\begin{array}{l}\text { Daphnia magna Straus,1820 * } \\
\text { Daphnia magna Straus,1820 }\end{array}$ & 0.38 & 96 & neonato \\
$\begin{array}{l}\text { Daphnia magna Straus,1820 } \\
\text { Daphnia magna Straus,1820 }\end{array}$ & 2.33 & 72 & neonato \\
$\begin{array}{l}\text { Homarus gammarus Linnaeus, } \\
\text { 1758 }\end{array}$ & 10 & 48 & neonato \\
$\begin{array}{l}\text { Penaeus californiensis (Holmes, } \\
\text { 1900) }\end{array}$ & 75 & 24 & neonato \\
\hline
\end{tabular}

* = presente estudio.

\section{Antiparasitarios}

El producto leche de ojé presentó mayor toxicidad en $D$. magna según los valores de $C_{50}$ que el producto comercial Parasín. La diferente composición de ambos antihlemínticos naturales explicaría los resultados obtenidos. El producto comercial "Leche de ojé” está compuesto por dos plantas: leche de ojé y hierba buena. En cambio el producto comercial Parasin tiene en su composición tres plantas: semillas de zapallo, semillas de papaya y hierba buena. El látex leche de ojé tiene mayor toxicidad que las semillas del zapallo y de la papaya (Díaz et al., 2004; Hansson et al., 2005).

La toxicidad del albendazol está relacionada con su modo de acción como benzimidazólico, al actuar como un tóxico mitótico en la división celular que desagrega los cromosomas en las células hijas, produciendo aneupliodia, y a su parámetro de lipofilicidad (Oh et al., 2006). Se ha observado que el valor de $\mathrm{CL}_{50}$ obtenido en el presente estudio en $D$. magna para el benzimidazólico, albendazol es significativamente más alto que un estudio registrado para $D$. magna de 67.9 ug $\mathrm{L}^{-1}$ a 48 h de exposición (Oh et al., 2006). Sin embargo, las diferentes condiciones del ensayo como la preparación de la solución madre del albendazol (pureza 98\% de Sigma-Aldrich), el empleo del acetato al 5\% como solvente, y el punto final de observación en las pulgas de agua, la inmobilidad condicionarían los resultados comparativos (Oh et al., 2006). Con el fin de determinar el riesgo en el ambiente acuático del albendazol se uso el cociente de riesgo (CR) (Versteeg et al., 2005; Ankley et al., 2005), por ende se empleó el valor de PEC (Concentración ambiental predicha) para este producto químico para aguas superficiales, existente para los sistemas acuáticos de Alemania y Korea (Koschorreck et al., 2002; Oh et al., 2006) de 0.66 ug L${ }^{1}$, mostrando luego de la aplicación del CR, que divide el valor de PEC sobre el valor de $\mathrm{CL}_{50}$ agudo de $180.4 \mathrm{mg}$ $\mathrm{L}^{-1}$ multiplicado por un valor de 1000 para estudios agudos (EC, 2003), lo cual determina la ausencia de riesgo del albendazol en el ambiente acuático. Sin embargo, un refinamiento en la estimación de los valores de PEC para el sistema de aguas superficiales del Perú es requerido para definir más adecuadamente el riesgo del albendazol en estas aguas.

Para el praziquantel, se ha observado un valor de $\mathrm{CL}_{50-48 \mathrm{~h}}$ de $30.07 \mathrm{mg} \cdot \mathrm{L}^{-1}$, el cual es similar al obtenido para un estudio previo con $D$. magna $\left(\begin{array}{llll}\mathrm{CL}_{50} & = & \mathrm{mg} \cdot \mathrm{L}^{-1}\end{array}\right)$ (http://www.pesticideinfo.org/Detail_Chemical.jsp) . Se ha observado en peces valores de toxicidad para el praziquantel que fluctuán entre 13.4 a $13480 \mathrm{mg} \cdot \mathrm{L}^{-1}$, siendo Clarias gariepinus (Burchell 1822 y Pimephales promelas Rafinesque 1820, la especie íctica mas sensible y la menos sensible entre cuatro especies de peces, respectivamente (http://www.pesticideinfo.org/Detail_Chemical.jsp)

\section{Literatura citada}

Akdogan M., Tamer M.N., Cure E., Cure M.C., Koroglu B.K. \& Delibas N. 2007. Effect of spearmint (Mentha spicata Labiatae) teas on androgen levels in women with hirsutism. Phytotherapia Research 20: (In press).

Alvarez-Sánchez M.A., Mainar-Jaime R.C., Pérez-García J. \& Rojo-Vázquez F.A. 2006. Resistance of Fasciola hepatica to triclabendazole and albendazole in sheep in Spain. Veterinary Record. 159: 424-425. 
Andersen W.C., Turnipseed S.B. \& Roybal J.E. 2006. Quantitative and confirmatory analyses of malachite green and leucomalachite green residues in fish and shrimp. Journal of Agriculture and Food Chemical. 54: 4517-4523.

Ankley G.T., Black M.C., Garric J., Hutchinson T.H. \& Iguchi T. 2005. A framework for assessing the hazard of pharmaceutical materials to aquatic species. pages 183-238. en: Human pharmaceuticals assessing the impacts on the aquatic ecosystems. Williams, R.T. (ed.). Society of Environmental Toxicology and Chemistry (SETAC). Pensacola (Fl), USA.

Amorim C.Z., Marques A.D. \& Cordeiro R.S. 1991. Screening of the antimalarical activity of plants of the Cucurbitaceae family. Memorias do Instituto Oswaldo Cruz 86 Suppl. 2: 177-180.

Backhaus T., Scholze M. \& Grimme L.H. 2000. The single substance and mixture toxicity of quinolones to the bioluminescent bacterium Vibrio fisheri. Aquatic Toxicology. 49: 49-61.

Belkind-Valdovinos U., Belkind-Gerson J., Sánchez F.D., Espinoza R.M.M. \& Lazcano P.E. 2004. Nitazoxanide vs albendazole against intestinal parasites in a single dose and for three days. Salud Pública Méxicana. 46: 333-340.

Boxall A.B.A., Fogg L.A., Lindsay A.F., Kay P., Blakwell P.A., Pemberton E.J. \& Croxford A. 2003a. Priorization of veterinary medicines in the UK environment. Toxicology Letters. 142: 207-218.

Boxall A.B.A., Kolpin D.W. \& Halling-Sørensen B. 2003b. Are veterinary medicines causing environmental risk? Environmental Science and Technology. 37: 286A294A.

Boxall A.B.A. \& Long C. 2005. Veterinary medicines and the environment. Environmental Toxicology and Chemistry. 24: 759-760.

Castillo G. 2004. Ensayos toxicológicos y métodos de evaluación de calidad de aguas. Estandarización, intercalibración, resultados y aplicaciones. IMTA. México.

Chapman P.M. 2006. Emerging substances- emerging problems? Environmental Toxicology and Chemistry. 25: 1445-1447.

Christensen A.M., Ingersley F. \& Baun, A., 2006. Ecotoxicity of mixtures of antibiotics used in aquacultures. Environmental Toxicology and Chemistry. 25: 2208-2215.

Clifton J. $2^{\text {nd }}$ \& Leikin J.B. 2003. Methylene blue. American Journal of Therapeutics. 10: 289-291.

Costamagna S.R., Dupin J., Vaylet S. \& Pellegrino P. 2004. Evaluación del fijador-colorante azul de metileno para el diagnóstico directo de Tricomonas vaginalis. Acta Bioquímica Clínica Latinoamericana. 38: 307-309.

Daughton C.G. \& Ternes T.A. 1999. Pharmaceutical and personal care products in the environment: agents of subtle change? Environmental Health Perspectives. 107: 907-938.

Díaz O. D., Lloja L.L. \& Carvajal Z.V. 2004. Preclinical studies of Cucurbita maxima (pumpkin seeds) a tradicional intestinal antiparasitic in rural areas. Revista de Gastroenterología del Perú. 24: 323-327.

De Amorin A., Borba H.R., Carauta J.P., Lopes D., Kaplan M.A. 1999. Anthelmintic activity of the latex of Ficus species. Journal of Ethnopharmacology . 64: 255-258.
European Commission (EC). 2003. Technical guidance document on risk assessment. EUR 20418 EN/2. Joint Research Centre, European Commision, Italy.

\section{GESAMP}

(IMO/FAO/UNESCOIOC/WMO/WHO/IAEA/UN/UNEP Joint group of experts on the scientific aspects of marine environmental Protection. 1997. Towards safe and effective use of chemicals in coastal aquaculture. Red.Stud.GESAMP. 65: 40

Giraldo J., Rojas J., Huamaní L., Espinoza S. \& Girio Z. 2006. Efecto antihelmíntico de Ficus antihelmintica Mart (Ojé) (Moraceae) y Cyclanthera pedata (L.) Schrad (Caigua) (Cucurbitaceae) sobre Hymenolepis nana (Siebold, 1852) (Cestoda: Hymenolepididae). Biologist (Lima). 4: 1-2.

Grant D.C., Papoutsoglou C. \& Saroglia Q. 2001. The control of chemicals used in aquaculture in Europe. Journal of Applied Ichthyology. 17: 173-180.

Guarrera P.M. 1999. Tradicional antihelmintic, antiparasitic and repellent uses of plants in Central Italy. Journal of Ethnopharmacology. 68: 183-192.

Halling-Sørensen B., Nor Nielsen S., Lanzky P.P.F., Ingerslev F., Lützhøft H.C. \& Jorgensen S.E. 1998. Occurrence, fate, and effects of pharmaceutical substances in the environment- A review. Chemosphere. 36: 357-393.

Hansson A., Zelada J.C. \& Noriega H.P. 2005. Reevaluation of risk with use of Ficus insipida latex as a traditional anthelmintic remedy in the Amazon. Journal of Ethnopharmacology. 98: 251-257.

Hempel H., Scheffczyk A., Schallnab H.J., Lumaret J.P., Alvinerie M. \& Römbke J. 2006. Toxicity of four veterinary parasiticides on larvae of the dung beetle Aphodius constans in the laboratory. Environmental Toxicology and Chemistry. 25: 3155-3163.

Hernando M.D., De Vettori S., Martinez B.M.J. \& Fernandez A.A.R. 2007. Toxicity evaluation with Vibrio fisheri test of organic chemicals used in aquaculture. Chemosphere. 8: (In press).

Holm-Martin M., Levot G.W. \& Dawson K.L. 2005. Control of endoparasites in horses with a gel containing moxidectin and praziquantel. Veterinary Record. 156: 835-838.

Iannacone J., Alvariño L., Soto J.C. \& Salcedo C. 2007. Efecto toxicológico del Sachayoco, Paullinia clavigera (Sapindaceae) sobre Daphnia magna y sobre dos controladores biológicos de Plagas agrícolas. Journal of the Brazilian Society of Ecotoxicology. 2: 15-25.

Iannacone J., Caballero C.R. \& Renteria J.A. 1999. La técnica de precoloración de Walker para evaluar Plasmodium vivax Grassi y Plasmodium malariae Laveran en comunidades asháninkas en Satipo (Junín, Perú). Revista peruana de Biología. 6: 171-180.

Imai H., Osawa K., Yasuda H., Hamashima H., Arai T. \& Sasatsu M. 2001. Inhibition by the essencial oils of peppermint and spearmint of the growth of pathogenic bacteria. Microbios. 106 Suppl: 31-39.

Jjemba P.K. 2006. Excretion and ecotoxicity of pharmaceutical and personal care products in the environment. Ecotoxicology and Environmental Safety. 63: 113-130.

Kallquist T., Grung M. \& Tollefsen K.E. 2006. Chronic toxicity of $2,4,2^{\prime}, 4^{\prime}$-Tetrabromodiphenil ether on the marine alga Skeletonema costatum and the crustacean 
Daphnia magna. Environmental Toxicology and Chemistry. 25: 1657-1662.

Karanja D.M.S., Boyer A.E., Strand M., Colley D.G., Nahlen B.L., Ouma J.H. \& Secor W.E. 1998. Studies on schistosomiasis in Western Kenya: II. Efficacy of praziquantel for treatment of Schistosomiasis in persons coinfected with human immunodeficiency virus-1. American Journal of Tropical Medicine and Hygiene. 59: 307-311.

Kim K.H., Lee E.H., Kwon S.R. \& Cho J.B. 2001. Treatment of Microcotyle sebastis infestation in culture rockfish Sebastes schlegeli by oral administration of praziquantel in combination with cimetidine. Disease of Aquatic Organisms. 44: 133-136.

Klüttgen B., Dülmer U., Engels M. \& Ratte H.T. 1994. $\mathrm{ADaM}$, an artificial freshwater for culture of zooplankton. Water Research. 28: 743-746.

Koschorreck J., Koch C. \& Ronnefahrt I. 2002. Environmental risk assessment of veterinary medicinal products in the EU- a regulatory perspective. Toxicology Letters. 131: 117-124.

Lagarto P.A., Tillán C.J., Vega M.R. \& Cabrera G.Y. 1999. Toxicidad aguda oral de extractos hidroalcohólicos de plantas medicinales. Revista Cubana de Plantas Medicinales. 1: 26-28.

Lalumera G.M., Calamari D., Galli P., Castiglioni S., Crosa G. \& Fanelli R. 2004. Preliminary investigation on the environment occurence and effects of antibiotics used in aquaculture in Italy. Chemosphere. 54: 661-668.

Leathwick D.M., Miller C.M., Atkinson D.S., Haack N.A., Alexander R.A., Oliver A.M., Waghorn T.S., Potter J.F. \& Sutherland I.A. 2006. Drenching adult ewes: implications of anthelmintic treatments pre- and postlambing on the development of anthelmintic resistance. New Zealand Veterinary Journal. 54: 297-304.

Lützhøft H.C.H., Halling-Sørensen B. \& Jørgensen S.E. 1999. Algal toxicity of antibacterial agents applied in Danish fish farming. Archives of Environmental Contamination and Toxicology. 36: 1-6.

Macedo M.E., Consoli R.A., Grande T.S., Dos Anjos A.M., De Oliveira A.B., Mendes N.M., Queiroz R.O. \& Zani C.L. 1997. Screening of Asteraceae (Compositae) plant extracts for larvicidal activitry against Aedes fluviatilis (Diptera: Culicidae). Memórias do Instituto Oswaldo Cruz. 92: 565-570.

Maguiña A.A. \& Iannacone J.A. 2000. Artemia franciscana Kellog 1906 “Camarón salino” como agente de bioensayo para evaluar cinco extractos crudos de plantas propiedades antiparasitarias. Boletín de la Sociedad Química del Perú. 66: 154-169.

Molnar K. 1995. Effect of exposure to malachite green solution on common carp fry Dactylogyrus vastator (Monogenea) infection. Acta Veterinaria Hungarica .43: 277-286.

Moreno H.A., Droppeiman C.V. \& Verdejo M.E. 2006. Evaluación de carbón activado producido a partir de lodo generado en una planta de tratamiento de aguas servidas. Información tecnológica. 17: 9-14.

Oh S.J., Park J., Lee M.J., Park S.Y., Lee J.H. \& Choi K. 2006. Ecological hazard assessment of major veterinary benzimidazoles: acute and chronic toxicities to aquatic microbes and invertebrates. Environmental Toxicology and Chemistry. 25: 2221-2226.
Pareja R.R., Leucona B.G. \& Ciriza J.A.F. 2002. Cefalea en un paciente joven. Neurocisticercosis. Medifam. 12: 650-654.

Reynaldi S., Duquesne S., Jung K. \& Liess M. 2006. Linking feeding activity and maturation of Daphnia magna following short-term exposure to fenvalerate. Environmental Toxicology and Chemistry. 25: 18311835.

Rifici L.M., Cherry D.S., Farris J.L. \& Cairms J.Jr. 1996. Acute and subchronic toxicity of methylene blue to larval fathead minnows (Pimephales promelas): implications for acuatic toxicity testing. Environmental Toxicology and Chemistry. 15: 1304-1308.

Rintamaki K.P., Rahkonen M., Mykra H. \& Valtonen E.T. 2005. Treatment of ichthyopthiriasis after malachite green. II. Earth ponds at salmonid farms. Disease of Aquatic Organisms. 66: 15-20.

Schmahl G., Ruider S., Mehlhorn H., Schmidt H. \& Ritter G. 1992. Treatment of fish parasites. 9. Effects of a medicated food containing malachite green on Ichthyophthirius multifiliis Fouquet 1876 (Hymenostomatida, Ciliophora) in an ornamental fish. Parasitology Research. 78: 183-192.

Sibat H.F. \& Ibañez V.I. 2003. Vascular dementia type binswanger's disease in patients with active neurocysticercosis. Electronic Journal of Biomedicine. 1: $32-42$.

Silveira C.R., Prieto T.A. \& Ascencio V.F. 2004. Effects of different stressors in haematological variables in cultured Oreochromis aureus S. Comparative Biochemical and Physiology Part C Toxicology and Pharmacology. 139: 245-250.

Srivastava S., Sinha R. \& Roy D. 2004. Toxicological effects of malachite green. Aquatic Toxicology. 66: 319-329.

Stocche R.M., García L.V., dos Reis M.P., Klamt J.G. \& Évora P.R.B. 2004. Methylene blue to treat anaphylaxis during anesthesia. Case Report. Revista Brasileira de Anestesiologia. 54: 809-814.

Stockert J.C. \& Herkovits J. 2003. Photodynamic toxicity and its prevention agents in Bufo arenarum embryos. Toxicology. 192: 211-218.

Tripathi A.K., Prajapati V., Aggarwal A. K.K. \& Khanuja S.P. 2004. Piperitenone oxide as toxic, repellent, and reproduction retardant malarial vector Anopheles stephenesi (Diptera: Anophelinae). Journal of Medical Entomology. 41: 691-698.

Van de Riet J.M., Murphy C.J., Pearse J.N., Potter R.A. \& Burns B.G. 2005. Determination of malachite green and leucomalachite green in a variety of aquaculture products by liquid chromatography with tandem mass spectrometry detection. Journal of AOAC International. 88: 744-749.

Versteeg D.J., Alder A.C., Cunningham V.L., Kolpin D.W., Murray-Smith R. \& Ternes T. 2005. Environmental exposure modeling and monitoring of humans pharmaceutical concentrations in the environment. pages 71-110. en: Human pharmaceuticals assessing the impacts on the aquatic ecosystems. Williams, R.T. (ed.). Society of Environmental Toxicology and Chemistry (SETAC). Pensacola (Fl), USA.

Watt B.E., Proudfoot A.T., Bradberry S.M. \& Vale J.A. 2005. Poisoning due to urea herbicides. Toxicological Reviews. 24: 161-166.

Waranusantigui P., Pokethitiyook P., Kruatrachue M. \& Upatham E.S. 2003. Kinetic of basic dye (methylene blue) 
biosorption by giant duckweed (Spirodela polyrrhiza). Environmental Pollution. 125: 385-392.

Wollenberger L., Halling-Sørensen B. \& Kusk K.O. 2000. Acute and chronic toxicity of veterinary antibiotics to Daphnia magna. Chemosphere. 40: 723-730.

Woodward K.N. 2006. Veterinary pharmacovigilance. Part 3. Adverse effects of veterinary medicinal products in animals and on the environment. Journal of Veterinary Pharmacology Therapethics. 28: 171-184.
Yu T.W., Xu M. \& Dashwood R.H. 2004. Antimutagenic activity of spearmint. Environmental and Molecular Mutagenesis. 44: 387-393.

Zilberg D. \& Tamar S. 2006. Optimization and validation of a colorimetric assay for Tetrahymena sp. survival. Research in Microbiology. 157: 355-35.

\section{Apéndice de tablas}

Tabla 1. Valores comparativos de $\mathrm{CL}_{50}$ con especies de crustáceos acuáticos expuestos al verde de malaquita.

\begin{tabular}{|c|c|c|c|}
\hline Especie de crustáceo & $\begin{array}{c}\mathrm{CL}_{50} \\
\left(\mathrm{mg} \mathrm{L}^{-1}\right)\end{array}$ & $\begin{array}{c}\text { Tiempo de } \\
\text { exposición (h) }\end{array}$ & Estadio \\
\hline Penaeus monodon Fabricius 1798 & 0.012 & 96 & Zoea \\
\hline Penaeus stylirostris Stimpson 1874 & 0.016 & 24 & Nauplio \\
\hline Penaeus monodon Fabricius 1798 & 0.018 & 72 & Zoea \\
\hline Cancer magister Dana 1852 & 0.02 & 96 & Zoea \\
\hline Penaeus monodon Fabricius 1798 & 0.03 & 48 & Zoea \\
\hline Cancer magister Dana 1852 & 0.04 & 96 & Zoea \\
\hline Penaeus monodon Fabricius 1798 & 0.06 & 96 & Postlarva \\
\hline Penaeus monodon Fabricius 1798 & 0.07 & 72 & Postlarva \\
\hline Penaeus monodon Fabricius 1798 & 0.11 & 24 & Zoea \\
\hline Cancer magister Dana 1852 & 0.12 & 48 & Zoea \\
\hline Penaeus monodon Fabricius 1798 & 0.13 & 96 & Mysis \\
\hline Cancer magister Dana 1852 & 0.16 & 48 & Zoea \\
\hline Penaeus semisulcatus de Haan 1844 & 0.2 & 24 & Postlarva \\
\hline Penaeus penicillatus Alcock 1905 & 0.3 & 24 & Zoea \\
\hline Penaeus monodon Fabricius 1798 & 0.33 & 48 & Postlarva \\
\hline Penaeus monodon Fabricius 1798 & 0.61 & 72 & Mysis \\
\hline Penaeus duorarum Burkenroad 1939 & 0.64 & 96 & NI \\
\hline Penaeus monodon Fabricius 1798 & 1 & 48 & Mysis \\
\hline Macrobrachium rosenbergii (de Man 1879) & 1.14 & 24 & Zoea \\
\hline Penaeus japonicus (Bate 1881) & 1.18 & 24 & Zoea \\
\hline Palaemonetes kadiakensis Rathbun 1902 & 1.9 & 96 & Zoea \\
\hline Penaeus monodon Fabricius 1798 & 2.5 & 24 & Mysis \\
\hline Penaeus monodon Fabricius 1798 & 2.51 & 24 & Postlarva \\
\hline Penaeus monodon Fabricius 1798 & 2.9 & 24 & Postlarva \\
\hline Daphnia magna Straus 1820 * & 2.97 & 96 & Neonatos \\
\hline Cypridiopsis sp. & 3.45 & 96 & NI \\
\hline Metapenaeus ensis (De Haan 1844) & 3.73 & 24 & Zoea \\
\hline
\end{tabular}


Argulus sp.

Cypridiopsis sp.

Cypridiopsis sp.

Palaemonetes kadiakensis Rathbun 1902

Penaeus schmitti Burkenroad 1936

Penaeus japonicus (Bate 1881)

Homarus americanus H. Milne Edwards 1837

Homarus gammarus Linnaeus 1758

Macrobrachium rosenbergii (de Man 1879)

Penaeus schmitti Burkenroad 1936

Metapenaeus ensis (De Haan 1844)

Penaeus schmitti Burkenroad 1936

Penaeus schmitti Burkenroad 1936

$\mathrm{NI}=$ No indicado. $*$ = presente estudio.

$\begin{array}{ccc}5 & \text { NI } & \text { NI } \\ 5.86 & 6 & \text { NI } \\ 5.86 & 24 & \text { NI } \\ 9.1 & 24 & \text { Zoea } \\ 10 & 24 & \text { Postlarva } \\ 10.13 & 24 & \text { Postlarva } \\ 20 & 1 & 4 \text { to juvenil } \\ 20 & 1 & 4 \text { to juvenil } \\ 21 & 24 & \text { Postlarva } \\ 46 & 72 & \text { Zoea } \\ 61 & 24 & \text { Postlarva } \\ 77 & 48 & \text { Zoea } \\ 323 & 24 & \text { Zoea }\end{array}$

\footnotetext{
${ }^{1}$ Facultad de Ciencias Biológicas, Universidad Particular Ricardo Palma. E-mail: joseiannacone@yahoo.es

2 Laboratorio de Ecofisiología Animal. Facultad de Ciencias Naturales y Matemáticas. Universidad Nacional Federico Villarreal. Calle San Marcos 383, Pueblo Libre, Lima, Perú.
} 